\title{
Designing scheme for Portable ECG System with Real-Time Application
}

\author{
P M Dudhat ${ }^{1}$, N P Joshi ${ }^{2}$, D S Pipalia ${ }^{3}$ \\ ${ }_{1,2,3}$ (Electronics and Communication Department, RK. University, India)
}

\begin{abstract}
Portable ECG system is used for long term monitoring of patients at the risk of life threatening cardiac arrhythmias. The idea of design work is to develop an algorithm for efficient QRS detection with low power consumption. The design will use OMAP 3530 application based processor and DSP core for ECG signal conditioning. After processing data, it will send to the clinical station through USB dongle or over the internet. The QRS detection algorithm uses matched filter to achieve optimized filtering and high SNR. This electronic document justifies the application along with designing scheme.
\end{abstract}

Keywords - Cardiac Arrhythmias, ECG, OMAP, QRS detection

\section{INTRODUCTION}

Nowadays Aging societies are beginning to face the severe challenge of maintaining their health level with increasing costs of state-of-the-art treatments for a growing number of old patients. Providing affordable and high-level care is one of the biggest issues in public health care systems today. The older generation has never before had such huge amounts of personal income at its disposal, willing to track their personal well being and health beyond governmental support. Also, a growing number of life-style oriented individuals are beginning to use medical monitoring devices for recreational use in sports and daily life. Both aspects of the current societal situation results into one technological conclusion: a demand for mobile, yet powerful monitoring systems exists, serving both the patient in need by maintaining qualified but remote supervision. Both aspects particularly require the acquisition, fusion and analysis of a large number of physiological parameters by a light-weight, portable and power-saving device that is able to give qualified and autonomous warnings or suggestions to improve the level of fitness of its bearer. In other words, personalized health monitoring systems might in the future become as ubiquitous as current cell phones are today[1].

Portable ECG is a hand-handled device that helps to monitor heart stroke rehabilitation patients, postheart surgery patients or patients with critical heart disorder. Post heart surgery or post heart attack patients are kept under walking or aerobic exercise rehab. It is proven that this rehab reduces recurrent heart stroke by $17 \%$ and thus avoids re-hospitalization and even deaths in certain cases. During rehab period, it is very vital to monitor ECG of patients as it give precise state and condition of recovery in heart.

In past, Holter devices are used to record ECG waveform. It is a portable device which is used for continuous monitoring of various electrical activity of the cardiovascular system for atleast 24 hours (often for 2 weeks at a time). But the main disadvantage of Holter device is that it only records serious rhythm irregularity of ECG signal and making it possible for later diagnosis but it is not react in real time [2]-[4].

Nowadays Arrhythmia monitors are used for ambulatory patients which analyze the ECG data in real time. In this, high end of processors like OMAP with DSP core are used to do signal processing and send data over wireless link to central station where a physician can interpret it. Such devices require very precise recognition of QRS complex and any false detection will cause misinterpretation. This system will give high accuracy and less power consumption.

In ECG data, QRS detection is most difficult task because of various noises present in the QRS complex. The noise sources are muscle noise, power- line interference, artifacts due to electrode motion. These noises can be removed by performing several operations on the data like filtering, differentiation, squaring and time-averaging of the signal.

After processing the data, USB dongle is used to send ECG data to a remote health care center through wireless communication network, where the signals are analyzed by physicians. It can also stream live ECG over internet. Again, this dongle helps doctor to login into portable ECG device remotely and read the data. Thus, wireless USB dongle established connectivity of mini ECG with internet and thus making it accessible for remote monitoring. 


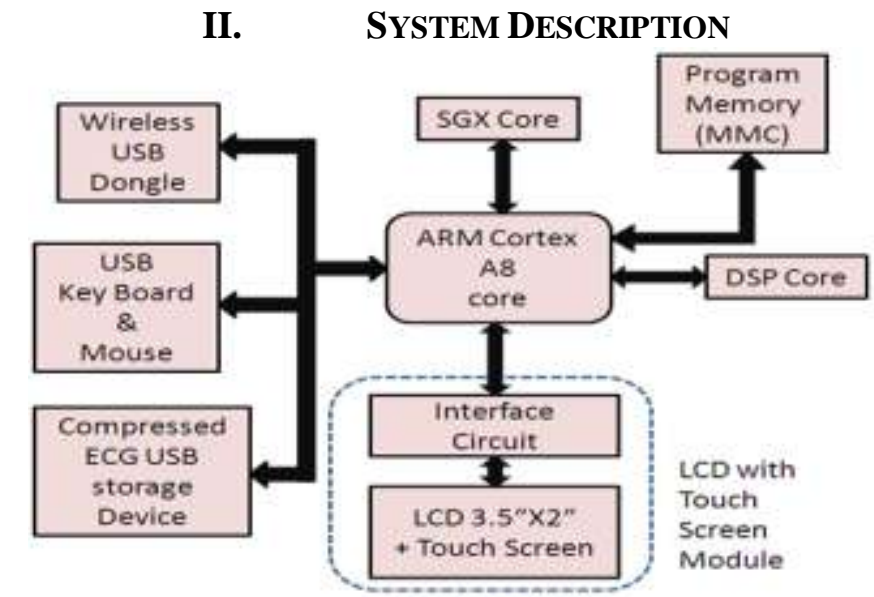

Figure 1 Portable ECG system with real time application

The portable ECG system consists of ARM cortex A8 processor which is the heart of the device and performs operation like I/O, storage, display and transmission. MMC is used as program memory. Wireless USB dongle, keyboard, mouse, LCD with touch screen and USB mass storage device are the other hardware peripherals. A powerful DSP core is used in the system which is OMAP 3530 application based processor which is used to do signal conditioning on the ECG data. It performs the QRS detection algorithm based on matched filter on the ECG data to remove noises. After signal processing, the mini ECG can transmit the stored data over internet through wireless USB dongle. It can also transmit over internet. This dongle helps doctor to login into mini ECG remotely and read the real-time data. Thus, wireless USB dongle established connectivity of mini ECG with internet and thus making it accessible for remote monitoring. An external USB storage device is used to store the compressed ECG data. User can read this device and data can be display on the LCD display. Before storing the ARM core with help of DSP core will perform compression on the ECG data and then dump that compressed data into the USB memory. The compression algorithm is activated whenever a recorded ECG signal is asked to be stored. USB Storage device is also used while ECG data is just getting recorded, once recording stops, ARM core reads the start of recording and runs compression algorithm and then again dumps the data back to the USB storage device. Touch screen provides user friendly interface to control mini ECG. As device is hand handled it is difficult to provide input through mouse or keyboard and hence, touch screen becomes mandatory. Interface circuit of touch screen reads the analog voltage for equivalent X-Y position and converts to digital. LCD screen is the main display of the device [11].

\section{II.I Beagleboard - An OMAP Based System}

The BeagleBoard is designed specifically to address the Open Source Community and has freely supported operating system developed by Texas Instruments(TI) coming with a growing repository of working applications. The large and advantageous development community of BeagleBoard developers can often solve a problem faster than the technical support can do, although most community efforts are currently still aimed towards the embedded linux ARM part of the system. Questions about pure DSP programming may still be better guided at TIs official support[1].

On top of the lower price and larger and faster support availability, the BeagleBoard has another advantage: Its physical dimensions are just around $10 \times 10 \mathrm{~cm}$ which is more suitable for portable applications. The USB-powered BeagleBoard is a low-cost, fan-less single board computer based on a TI OMAP3730CBP applications processor that is said to reach laptop-like performance and integrates a $1 \mathrm{GHz} \mathrm{ARM}$ Cortex-A8 core with a high-end $800 \mathrm{MHz}$ DSP-TMS320C64x core. The OMAP3530 supports high-level operating systems (OSs), such as Windows CE, Linux and others. As can be seen in Fig. 2, a USB OTG (On-the-Go) port exists on the BeagleBoard and has two modes: in client mode, the USB port lets the beagle board act as a common USB device that can be connected to a PC. In host mode, the beagle board becomes the main device and can use other USB devices as clients. USB mice, keyboards, network interfaces, and hard disks can be connected to the beagle board in this way. Besides new specialized fast SPI ports (McSPI) on the OMAP3530, there are also five McBSP ports present. McBSP2 provides a full-duplex, direct serial interface between an audio codec module inside the TWL4030 chipset. The video interface of the OMAP3530 is accessible through a DVI-D interface connector located on the board. The BeagleBoard contains JTAG, RS232, SVideo and expansion ports for different applications. Additional hardware can easily be connected via USB as mentioned above[5]. 


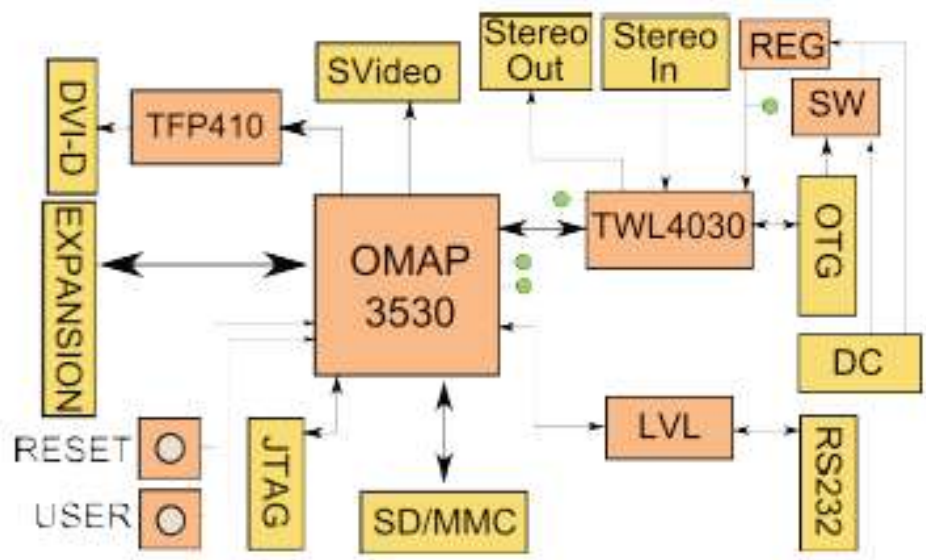

Figure 2 A BeagleBoard layout

III. PROPOSED METHOD

An ECG represents the electrical signal moving through the heart during a heartbeat. The signal's movement is divided into parts, and each part is given an alphabetic designation in the ECG Fig. 3 [6]. In this P wave represents atrial depolarization. It occurs when the sinoatrial (SA) node creates an action potential that depolarizes the atria. The QRS complex comes after the $\mathrm{P}$ wave which indicates the SA nodal action potential travels through AV (atrioventrical) node to ventricles to cause ventricle depolarization. The $\mathrm{T}$ wave comes after QRS complex and it is a result of ventricular repolarization.

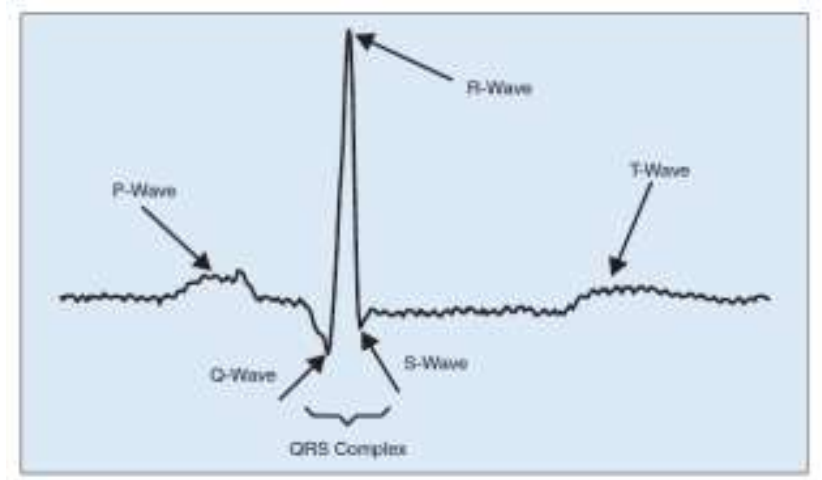

Figure 3 QRS complex of ECG

\section{III.I QRS Complex Detecting Algorithm}

As QRS complex is difficult in ECG data, it is necessary to detect the QRS complex without noise. So to detect the noises in ECG data, different algorithms are used which are software QRS detection [6], Filter banks [7], wavelet transform [8], Principle Component Analysis (PCA) [9] and Adaptive Compressive Algorithm [10].

Pan and Tompkins have developed a real time QRS detection algorithm that efficiently detects the QRS complex using digital analysis of slope, amplitude and width information of ECG waveforms. The real time QRS detection algorithm includes three different steps: linear digital filtering, non- linear transformation and decision rule algorithms. According to these processing steps, Pan and Tompkins method have performed linear processing using a band pass filter (low pass filter and high pass filter), a derivative and moving window integrator. The nonlinear transformation performed by the algorithm is signal amplitude squaring. Adaptive threshold and $\mathrm{T}$ wave discrimination techniques are used as a part of the decision rules for reliable QRS detection[11]. 


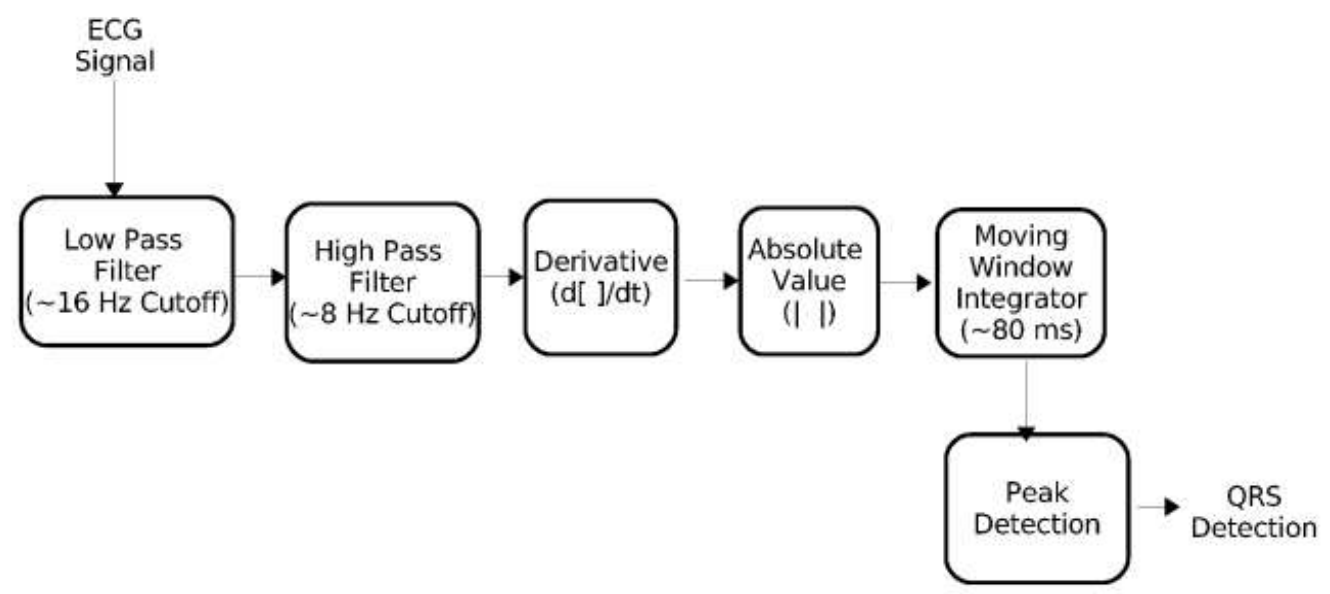

Figure 4 Filter stages for QRS detection algorithm [11]

Fig 4 shows the algorithm for noise removal in QRS detection. This algorithm is divided into two steps: filtering and decision rules. The aim of filtering of the signal is to generate a windowed estimate of the energy in the QRS frequency band. That is achieved using following tasks: (1) low pass filter of cutoff frequency $\sim 16 \mathrm{~Hz}$ and delay $\sim 20 \mathrm{~ms}$ (2) high pass filter of cutoff frequency $\sim 8 \mathrm{~Hz}$ and delay $\sim 60 \mathrm{~ms}$, (3) derivative, to extract slope information and delay $\sim 5 \mathrm{~ms}$, (4) absolute value function (5) moving window integrator of size $\sim 80 \mathrm{~ms}$. After filtering the signal is free from muscle noise, $50 / 60 \mathrm{~Hz}$ power line interference, baseline wander and motion artifacts. The decision rules are used to discriminate the QRS events from the noise events[11].

Each of the stages in this QRS detection technique is explained in the following sections. In this report sampled ECG signal is used with the reference databases (such as AHA and MIT/BIH) that will serve as an example input signal for the processing steps to follow.

\section{III.I.I Band Pass Filtering}

The first step in the QRS detection algorithm is the band pass filtering which is the combination of low pass filtering and high passes filtering. The recurrence formula for band pass filter is given by,

$y_{n}=1.875 * y_{n-1}-0.9219 * y_{n-2}+x_{n} * x_{n-2}$

which is implemented using the equivalent time domain difference equation,

$y(n T)=1.875 y(n T-T)-0.9219 y(n T-2 T)+x(n T)-x(n T-2 T)$

\section{III.I.II Derivative}

After the signal has been filtered out, it is then differentiated to provide information about the slope of QRS detection. The derivative approximation is implemented using following time domain difference equation,

$y(n T)=\frac{2 x(n T)+x(n T-T)-x(n T-3 T)-2 x(n T-4 T)}{8}$

III.I.III Absolute Function

Once the slope information is known, absolute value of each sample is captured using the operation,

$y(n T)=|x(n T)|$

This function will make all the data points to be positive and improve gain sensitivity of QRS detector which will improve performance of algorithm. The output of this stage should be limited to certain maximum level corresponding to the number of this bits used to represent the data type of the signal.

\section{III.I.IV Moving Window Integral}

The purpose of moving window integrator is to capture the prominent waveforms of ECG signal. The selection of size of window is very dominant during the implementation of window detector. If the size of window is too small then some of the complexes may produce several peaks in the integration waveform. If the 
size of window is too large, then integration waveform will merge the QRS complex and T waveform together. So it is necessary to choose size of window properly which is nearer to the width of a typical QRS complex. (e.g, less than $100 \mathrm{~ms}$ ). In this work the average size of window is chosen $80 \mathrm{~ms}$. It is implemented with the following difference equation,

$y(n T)=\frac{x(n T-N-1) T)+x(n T-(N-2) T)+\cdots+x(n T)}{N}$

Where $\mathrm{N}$ is the number of samples in the width of moving window.

\section{III.I.V Decision Rules}

After the preprocessing, the signal is tested on decision rules. The role of decision rules is to differentiate between QRS complex and noise level.

\section{Peak Detection}

The peak detector locates the peaks of QRS complex in the filtered signal. The detected peak is defined as an event. Once a peak is detected it is classified as either a true value or a noise. The algorithm uses the peak height, peak location and maximum derivative to classify the peaks[11].

\section{Rules can be summarized as follows:}

Rule 1: All peaks that precede the larger peaks before $200 \mathrm{~ms}$ can be ignored.

Rule 2: If a peak occurs, then there is a need to check whether the raw signal contains both positive and negative slopes of nearly same magnitude in a $200 \mathrm{~ms}$ window. If it is not, then the peak represents the noise.

Rule 3: Peak can be detected in the regions where signal rises above the threshold. That peak is referred as a QRS complex, otherwise it is a noise.

Rule 4: If no QRS complex is detected within a time interval of $3 / 2 \mathrm{RR}$ intervals, then the peak greater than the $1 / 2$ of the threshold, and the peak followed by the previous detection at least $360 \mathrm{~ms}$, can be classify as a QRS complex.

\section{Threshold Estimation}

The threshold (v) used in Rule 3 and Rule 4 is estimated from QRS peak height ( $\mathrm{h}_{\mathrm{QRS}}$ ) and noise peak height $\left(\mathrm{h}_{\text {noise }}\right.$ ). Once a peak is classified as QRS peak it is stored in a buffer with the eight most recent QRS peaks. Similarly, if a peak is classified as noise peak, it is added to the noise buffer containing eight most recent noise peaks. The threshold is estimated to some value between the mean of the QRS peak height $\left(\mu \mathrm{h}_{\mathrm{QRS}}\right)$ and mean of noise peak height $\left(\mu \mathrm{h}_{\text {noise }}\right)$ buffers using the following formula,

$v=\mu_{\text {hnoise }}+C_{\mathrm{v}}\left(\mu_{\mathrm{hQRS}}-\mu_{\mathrm{hnoise}}\right)$

where $C_{\mathrm{v}}$ is the threshold coefficient that lies between 0 and 1[11].

IV. RESULTS

The following screen shots show the output from different stages of Fig 4.

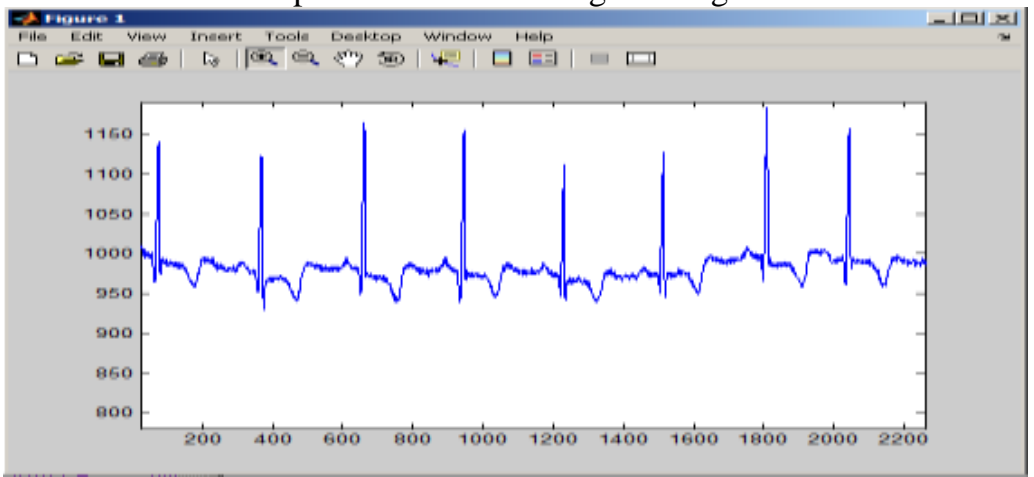

Figure $5 \mathrm{~A}$ recorded ECG signal of MIT/BIH using a test subject 


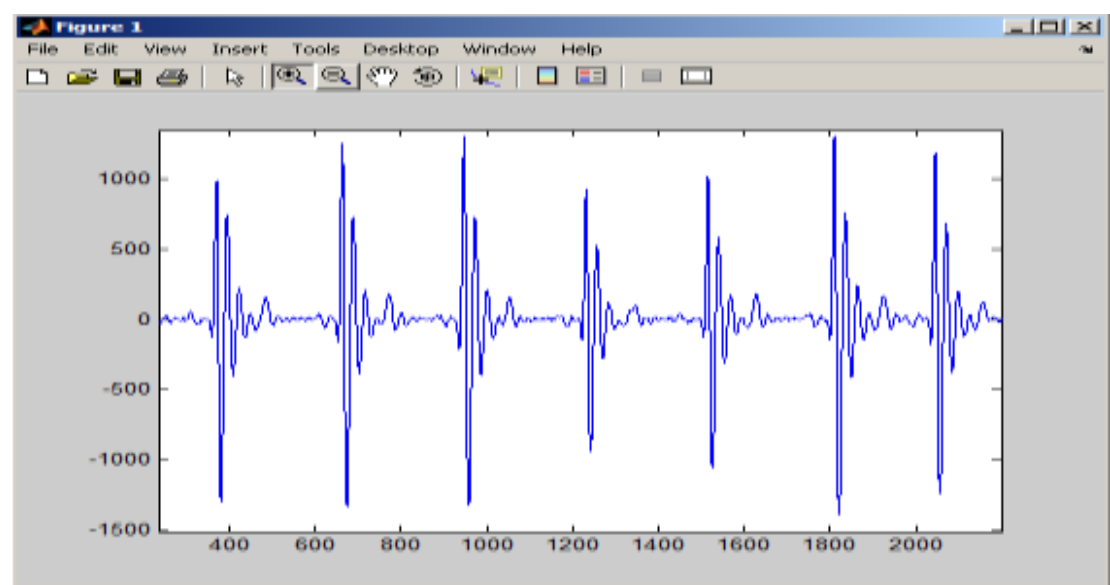

Figure 6 Band pass filtered result of noisy ECG signal

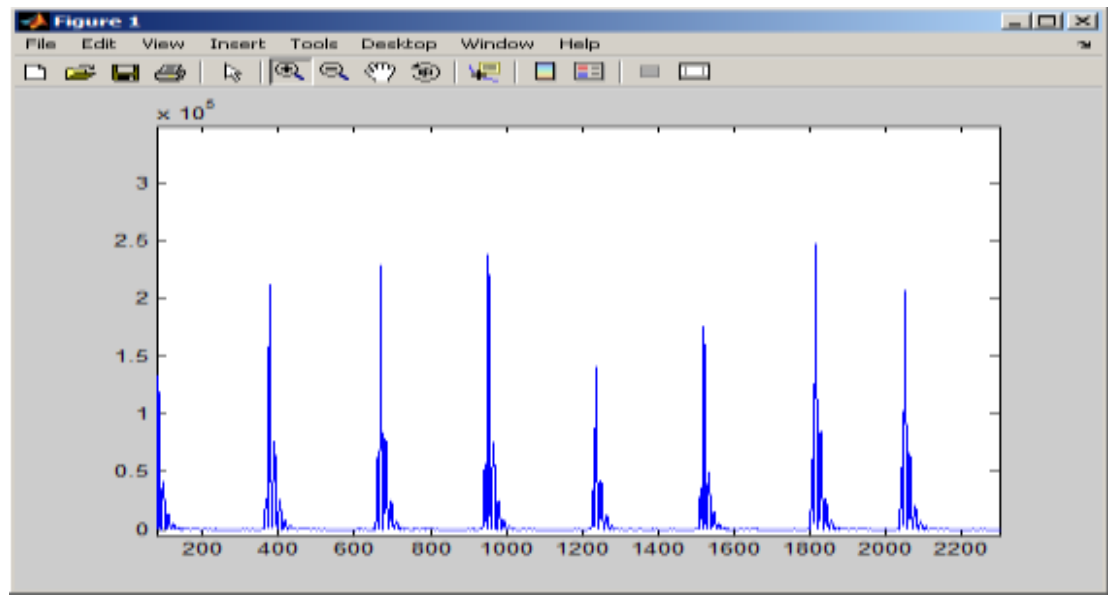

Figure 7 Derivative and Absolute result of ECG signal

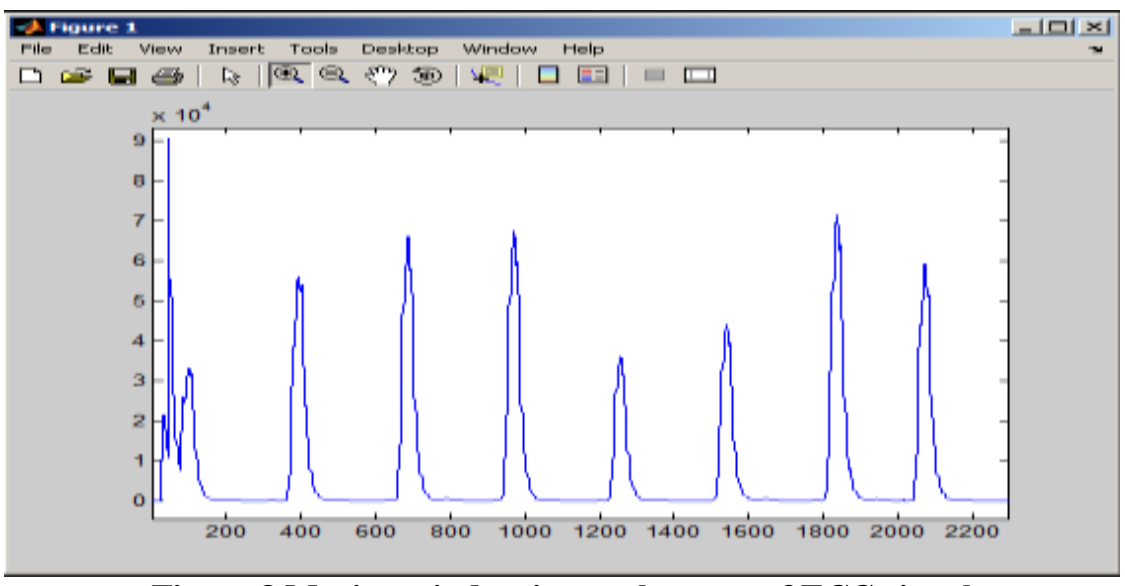

Figure 8 Moving window integral output of ECG signal

\section{CONCLUSION}

The QRS detection algorithm and hardware implementation for ECG monitoring with real time application are presented in this work. The QRS detection has been implemented with the use of band pass filter, derivative, absolute value and moving window integrator, which improve SNR. This will permit the use of thresholds that are above the noise peak level. Therefore, the overall sensitivity of the detector will improve. The decision has been done in real time. For implementation in real time, high end processor OMAP based on ARM Cortex A8 is used. It will give low cost, low power consumption patient monitoring in the lifethreatening events. 


\section{REFERENCES}

[1] K. Mankodiya, S.Vogt, A. Kundu, M. Klostermann, J. Pohl, A. Ayoub, H. Gehring, U. G. Holfmann "PORTABLE ELECTROPHYSIOLOGIC MONITORING BASED ON THE OMAP-FAMILY PROCESSOR FROM A BEGINNERS' PROSPECTIVE"

[2] R. Fensli, E. Gunnarson, O. Hejlesen. "A Wireless ECG System for Continuous Event Recording and Communication to a clinical alarm station", $26^{\text {th }}$ annual international Conference of IEEE EMBS, 2004.

[3] James Welch, Farzin Guilak, Steven D. Baker. "A Wireless ECG Smart Sensor for Broad Application in Life Threatening Event Detection", $26^{\text {th }}$ annual internation conference of IEEE EMBS, 2004

[4] Jimena Rodriguez, Lacramioara Dranca, Alfredo Goni and Arantza Illarramendi. "A web Access to Data in a Mobile ECG Monitoring System", University of Basque Country. LSI Department. Donosia-San Sebastian. Spain, http://siul02.si.ehu.es

[5] BB_SRM_xM 2010: BeagleBoard-xM System Reference Manual 2010

[6] Bert-Uwe Köhler, Carsten Hennig, Reinhold Orglmeister, "The Principles of Software QRS Detection", IEEE ENGINEERING IN MEDICINE AND BIOLOGY, January/ February 2002

[7] V. X. Afonso, W.J. Tompkins, T. Q. Nguyen, and S. Luo, “ECG beat detection using filter banks,” IEEE Trans. Biomed. Eng., vol. 46, pp. 192-201, Feb.1999

[8] Cuiwei Li, Chongxun Zheng, and Changfeng Tai, "Detection of ECG Characteristic Points Using Wavelet Transforms", IEEE TRANSACTIONS ON BIOMEDICAL ENGINEERING, VOL. 42, NO. 1, JANUARY 1995

[9] Philip Langley*, Emma J. Bowers, and Alan Murray, "Principal Component Analysis as a Tool for analyzing Beat-to-Beat Changes in ECG Features: Application to ECG-Derived Respiration”, IEEE TRANSACTIONS ON BIOMEDICAL ENGINEERING, VOL. 57, NO. 4, APRIL 2010

[10] Beatrice Arvinti*, Alexandru Isar** and Marius Costache*, "An Adaptive Compression Algorithm for ECG Signals", 12th IEEE International Symposium on Computational Intelligence and Informatics • 21-22 November, 2011

[11] http://www.physik.uni-freiburg.de/ severin/ECG_QRS_Detection.pdf Valtino X. Afonso, “ECG QRS Detection” Physikalisches Institute

[12] http://code.google.com/p/miniecg/ 